Case Report

\title{
Immune Thrombocytopenia in a Child with Neuroblastoma
}

\author{
Hasan Tarkan Ikizoglu, ${ }^{1}$ Inci Ayan, ${ }^{2}$ Fatma Tokat, ${ }^{3}$ \\ Tulay Tecimer, ${ }^{3}$ and Gonca Topuzlu Tekant ${ }^{4}$ \\ ${ }^{1}$ Department of Pediatrics, Acibadem University Medical Faculty, Istanbul, Turkey \\ ${ }^{2}$ Department of Pediatrics, Acibadem Maslak Hospital, Istanbul, Turkey \\ ${ }^{3}$ Department of Pathology, Acibadem Maslak Hospital, Istanbul, Turkey \\ ${ }^{4}$ Department of Pediatric Surgery, Cerrahpasa Medical Faculty, Istanbul University, Istanbul, Turkey \\ Correspondence should be addressed to Hasan Tarkan Ikizoglu; htarkani@gmail.com
}

Received 2 May 2017; Revised 1 July 2017; Accepted 13 August 2017; Published 14 September 2017

Academic Editor: Edvige Veneselli

Copyright (C) 2017 Hasan Tarkan Ikizoglu et al. This is an open access article distributed under the Creative Commons Attribution License, which permits unrestricted use, distribution, and reproduction in any medium, provided the original work is properly cited.

Thrombocytopenia is a frequent finding in patients with solid tumors. It is usually caused by bone marrow infiltration or by myelosuppression due to anticancer therapy; however immune thrombocytopenia (ITP) associated with solid tumors is rare. Neuroblastoma is the most common extracranial solid tumor in children. Here we report the case of a two-year-nine-monthold patient with adrenal neuroblastoma who presented with ITP. Paraneoplastic ITP was considered in the differential diagnosis. Bone marrow infiltration and other causes of thrombocytopenia were excluded and the patient was treated with intravenous immunoglobulin and tumor resection. Platelet count increased rapidly after surgery and complete remission of ITP was achieved.

\section{Introduction}

Thrombocytopenia is a frequent finding in patients with cancer and is usually caused by bone marrow involvement of malignant cells or by toxicity from anticancer therapy. Rarely immune thrombocytopenia (ITP) is reported at the time of diagnosis. Although this condition could represent incidental finding of two common pathologies, paraneoplastic processes should also be considered. Immune thrombocytopenia has been reported as a paraneoplastic syndrome with several tumors [1-3]. However, to our knowledge it has not been reported previously in patients with neuroblastoma.

Neuroblastoma is the most common extracranial solid tumor in children [4]. Neuroblastoma can arise anywhere along the sympathetic nervous system. The majority of tumors $(65 \%)$ arise in the abdomen, with over half of these arising in the adrenal gland. Clinical presentation of neuroblastoma is dependent upon the site of tumor origin, disease extent, and the presence of paraneoplastic syndromes [5].

Herein, we report a case of neuroblastoma presenting with ITP and we discuss the probability of paraneoplastic thrombocytopenia.

\section{Case Presentation}

A 2-year-9-month-old male patient with a left adrenal mass detected by abdominal ultrasonography was referred to our institution. His chief complaints were paleness, abdominal pain, and back pain of one-week duration. Fever or findings consistent with viral infection, history of vaccination, and drug use during the previous weeks were not reported. His previous medical history and family history were unremarkable.

He had mild pallor; his vital signs, growth, and development were normal. Physical examination revealed a mass at the left upper quadrant of the abdomen and several ecchymotic lesions measuring 1 to $1.5 \mathrm{~cm}$ in diameter, on the pretibial areas. Hepatosplenomegaly and lymphadenopathy were not detected. Complete blood count (CBC) revealed thrombocytopenia and mild anemia with hemoglobin $(\mathrm{Hb}): 9.6 \mathrm{~g} / \mathrm{dl}, \mathrm{MCV}$ : $75.8 \mathrm{fL}$, platelet (PLT): $53000 / \mathrm{mm}^{3}$, mean platelet volume (MPV): 9,1 fL, and white blood cell: $10740 / \mathrm{mm}^{3}$. Lactate dehydrogenase was $392 \mathrm{IU} / \mathrm{L}$ (normal range $\leq 345$ ); other laboratory tests including renal and liver function tests, serum electrolytes, prothrombin 


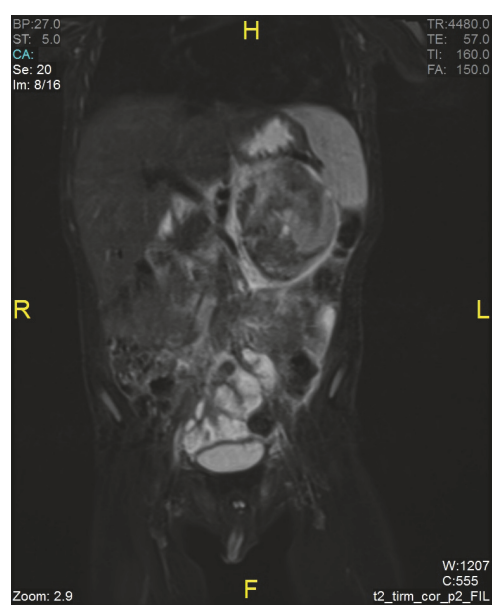

FIgURE 1: Heterogeneous mass at the left adrenal gland with smooth borders, containing cystic and hemorrhagic areas.

time, activated partial thromboplastin time, fibrinogen, and ferritin were within normal range. The serology tests for HIV, Hepatitis B, and Hepatitis $C$ were negative.

Abdominal MRI was performed. A heterogeneous mass with smooth borders, containing cystic and hemorrhagic areas, measuring $59 \times 60 \times 74 \mathrm{~mm}$ was detected at the left adrenal gland. The tumor did not cross the midline and no vascular invasion, lymphadenopathy, or metastases to other abdominal organs were detected with MRI (Figure 1). Neuroblastoma was considered.

Patient was admitted to hospital for further evaluation and surgery. Neuron specific enolase was found to be $26 \mathrm{ng} / \mathrm{ml}$ (normal range $\leq 20$ ); serum catecholamines were within normal range. Tumor metastasis was not observed in I-131 Metaiodobenzylguanidine and Technetium-99 m scan.

$\mathrm{CBC}$ was repeated and showed a platelet count of $26000 / \mathrm{mm}^{3}$. Bilateral bone marrow biopsy and aspiration were performed for tumor staging and for investigating the etiology of the thrombocytopenia. Cytomorphologic examination revealed normocellular bone marrow with continuous maturation in erythroid, myeloid, and megakaryocytic elements. The number of megakaryocytes was increased. Young forms of megakaryocytes and rare micromegakaryocytes were observed as well as mature forms. There were no tumor cells, pseudorosettes, storage cells, and/or erythrophagocytosis in the bone marrow; thus an immune thrombocytopenia was considered (Figure 2).

The patient was treated with $0.8 \mathrm{~g} / \mathrm{kg}$ of intravenous immunoglobulin (IVIG) and subsequently transfused with platelets in order to maintain an acceptable level of thrombocytes for diagnostic and therapeutic surgery [6]. An increase in platelet count was achieved from 26000 to $75000 / \mathrm{mm}^{3}$ at the third hour following IVIG and platelet transfusion. The patient underwent surgery and a $70 \times 65 \times 45 \mathrm{~mm}$ left adrenal mass was totally resected. The operation was completed without any major bleeding and/or other complications. Immediately after the surgery, platelet count was detected $96000 / \mathrm{mm}^{3}$; it was raised to normal levels at the

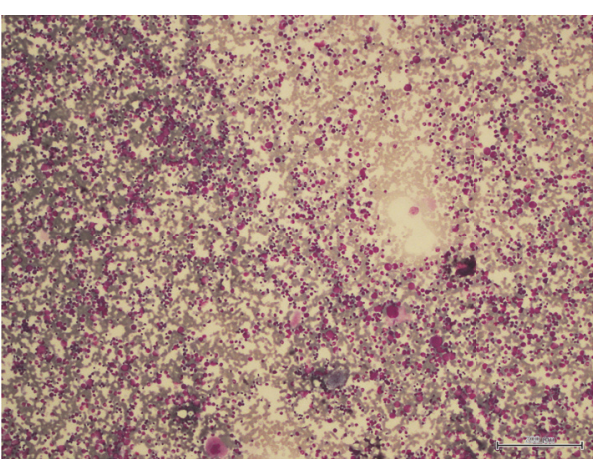

FIGURE 2: Normocellular heterogeneous bone marrow demonstrating increased number of mature and immature megakaryocytes.

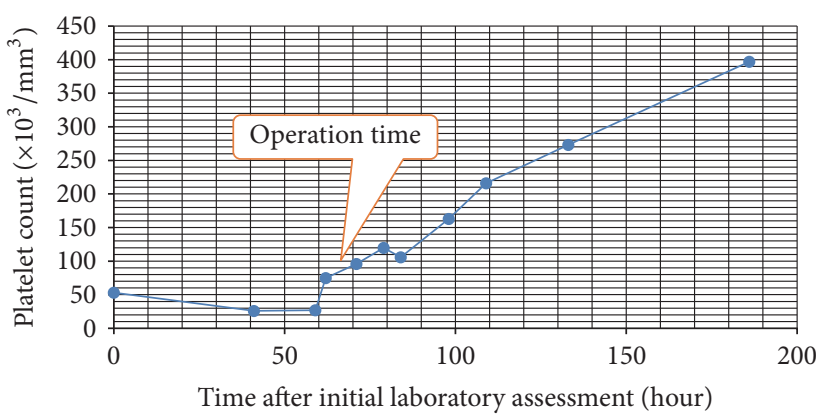

Figure 3: Platelet count during the follow-up.

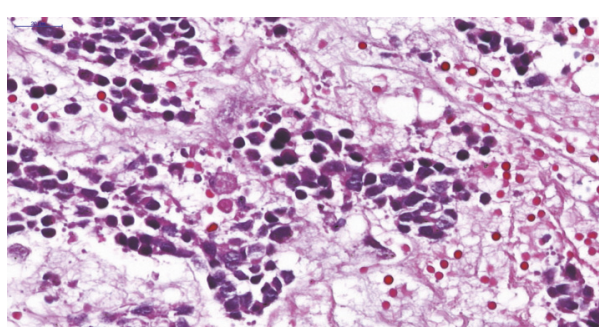

FIgURE 4: Neuroblastoma (Schwannian-stroma-poor), differentiating subtype, composed predominantly of differentiating neuroblasts with moderate to abundant acidophilic cytoplasm and enlarged, eccentric nucleus with vesicular chromatin and single prominent nucleolus. Abundant neuropil is also present.

36th hour after surgery and remained stable afterwards which supported the diagnosis of ITP (Figure 3).

Tumor histopathology revealed a Schwannian-stromapoor, differentiating neuroblastoma with low mitotic rate and low mitosis-karyorrhexis index (MKI < 100/5000 cells) [7] (Figure 4). The resection margins were free of tumor cells.

The patient was diagnosed as stage 1 neuroblastoma according to the International Staging System [8] and immune thrombocytopenia. According to the neuroblastoma treatment protocol the patient was not given any adjuvant chemotherapy and/or radiotherapy and a follow-up program was started. During the six-year follow-up recurrence of ITP was not observed. 


\section{Discussion}

Thrombocytopenia in cancer patients is induced by several mechanisms: (1) decrease in platelet production caused by bone marrow infiltration or by bone marrow suppression due to cytotoxic drugs, radiotherapy, or infections, (2) increased platelet destruction due to immune pathologies or disseminated intravascular coagulopathy (DIC), (3) platelet sequestration as a result of splenic metastasis. Immune thrombocytopenia is mainly a diagnosis of exclusion and all other causes for thrombocytopenia must be ruled out in order to confirm the diagnosis. ITP can be either primary (idiopathic) or secondary. Although ITP with tumors may be a coincidental finding, it has been described as a paraneoplastic syndrome of several tumors [1-3].

The association between ITP and lymphoid neoplasms, particularly chronic lymphoid leukemia and Hodgkin's disease, is well described $[1,9]$. The prevalence of ITP in Hodgkin's disease is $0.79 \%$ [1] and $0.76 \%$ in non-Hodgkin's lymphoma [10]. However, the association with solid tumors is less recognized. ITP has been reported to present with various types of solid tumors including breast, lung, kidney, germ cell, and ovary cancer [11].

Paraneoplastic syndromes are disorders that are not directly related to the growth, invasion, or metastasis of the tumor but occur as a result of autonomous secretion of hormones, peptides, or cytokines by the tumor. The major paraneoplastic syndromes associated with neuroblastomas are opsoclonus-myoclonus-ataxia syndrome (OMAS), which is believed to have an autoimmune pathogenesis $[4,12]$ and intractable watery diarrhea due to the autonomous tumor secretion of vasoactive intestinal peptide (VIP) $[4,13]$. To date, paraneoplastic ITP diagnosed at presentation in patients with neuroblastoma has not yet been reported.

In patients with neuroblastoma, although very frequent during chemotherapy and/or radiotherapy, thrombocytopenia at diagnosis is usually related to bone marrow infiltration by the tumor cells, which results in stage IV disease. In the present case, thrombocytopenia was detected at initial evaluation before the commencement of therapy. There was no evidence of infection or DIC. Splenic involvement that could result in thrombocyte sequestration was ruled out by abdominal MRI. Mean platelet volume was normal. Bilateral bone marrow aspiration and biopsy were performed and tumor involvement was ruled out. Bone marrow aspiration revealed normocellular bone marrow with no tumor cells, both young forms and mature megakaryocytes were observed and the number of megakaryocytes was increased suggesting an autoimmune process accompanying neuroblastoma rather than bone marrow infiltration. Although tests for platelet antibodies could not be performed we concluded that thrombocytopenia in this case was immunologic in origin after reviewing his clinical, laboratory, and imaging findings. Since ITP may occur in isolation (primary) or in association with other disorders (secondary), secondary causes were investigated [6]. The patient did not have fever or other findings consistent with viral infection during the initial examination or during the previous weeks. The use of any medication, recent vaccination, and blood transfusion were denied. HIV and hepatitis infection were ruled out with serological tests. There was no evidence of rheumatologic disease. Therefore we concluded that immune thrombocytopenia in this patient was either primary ITP or secondary ITP associated with neuroblastoma. Although neuroblastoma and primary ITP could occur coincidentally, rapid recovery of thrombocytopenia after tumor resection may support the diagnosis of paraneoplastic ITP.

The mechanism of immune thrombocytopenia associated with tumors remains to be elucidated. Immune dysregulation occurring in the setting of cancer is thought to result in increased platelet destruction [9]. Autoantibodies against platelet antigens have been demonstrated in some patients with ITP associated with lymphomas and solid tumors; however they were not shown to be specific for paraneoplastic ITP $[2,3,14,15]$.

Treatment of paraneoplastic ITP associated with solid tumors included various combinations of corticosteroids, splenectomy, IVIG, and interferon in addition to primary treatment of the underlying malignancy with surgery, chemotherapy, and/or radiotherapy. Complete remission of ITP after curative surgery was generally low and additional therapeutic agents were needed [11]. In the present case IVIG and subsequent platelet transfusion were used for managing ITP because rapid elevation of platelet count was essential for performing surgery. Platelet count was raised to normal levels after the resection of tumor without requiring any other supportive treatment.

In conclusion the aim of this case presentation is to emphasize that bone marrow infiltration is not the only cause of thrombocytopenia at diagnosis in neuroblastoma. Although rarely, an immune thrombocytopenia either primary or paraneoplastic has to be taken into consideration. A prompt and appropriate intervention for thrombocytopenia may allow the specific oncological treatment.

\section{Conflicts of Interest}

The authors declare that they have no conflicts of interest.

\section{References}

[1] K. Lechner and Y.-A. Chen, "Paraneoplastic autoimmune cytopenias in hodgkin lymphoma," Leukemia and Lymphoma, vol. 51, no. 3, pp. 469-471, 2010.

[2] K. Yang, T. Zhang, J. Chen et al., "Immune thrombocytopenia as a paraneoplastic syndrome in patients with nasopharyngeal cancer," Head and Neck, vol. 34, no. 1, pp. 127-130, 2012.

[3] S. Zheng, H. Chan, R. J. Epstein, and J. E. Joseph, "Resolution of paraneoplastic immune thrombocytopenia following everolimus treatment for metastatic renal cell carcinoma," Internal Medicine Journal, vol. 45, no. 6, pp. 666-669, 2015.

[4] P. E. Zage and J. L. Ater, "Neuroblastoma, in Nelson Textbook of Pediatrics," in Neuroblastoma, in Nelson Textbook of Pediatrics, R. M. Kliegman, Ed., pp. 2461-2464, Elsevier, Philadelphia, USA, 20th edition, 2016.

[5] J. R. Park, A. Eggert, and H. Caron, "Neuroblastoma: biology, prognosis, and treatment," Hematology/Oncology Clinics of North America, vol. 24, no. 1, pp. 65-86, 2010. 
[6] C. Neunert, W. Lim, M. Crowther, A. Cohen, L. Solberg Jr., and M. A. Crowther, "The American Society of Hematology 2011 evidence-based practice guideline for immune thrombocytopenia," Blood, vol. 117, no. 16, pp. 4190-4207, 2011.

[7] H. Shimada, I. M. Ambros, L. P. Dehner, J.-I. Hata, V. V. Joshi, and B. Roald, "Terminology and morphologic criteria of neuroblastic tumors: recommendations by the International Neuroblastoma Pathology Committee," Cancer, vol. 86, no. 2, pp. 349-363, 1999.

[8] G. M. Brodeur, J. Pritchard, F. Berthold et al., "Revisions of the international criteria for neuroblastoma diagnosis, staging, and response to treatment," Journal of Clinical Oncology, vol. 11, no. 8, pp. 1466-1477, 1993.

[9] E. Maverakis, H. Goodarzi, L. N. Wehrli, Y. Ono, and M. S. Garcia, "The etiology of paraneoplastic autoimmunity," Clinical Reviews in Allergy and Immunology, vol. 42, no. 2, pp. 135-144, 2012.

[10] A. W. Hauswirth, C. Skrabs, C. Schützinger et al., "Autoimmune thrombocytopenia in non-Hodgkin's lymphomas," Haematologica, vol. 93, no. 3, pp. 447-450, 2008.

[11] M.-T. Krauth, J. Puthenparambil, and K. Lechner, "Paraneoplastic autoimmune thrombocytopenia in solid tumors," Critical Reviews in Oncology/Hematology, vol. 81, no. 1, pp. 75-81, 2012.

[12] F. Blaes and B. Dharmalingam, "Childhood opsoclonusmyoclonus syndrome: diagnosis and treatment," Expert Review of Neurotherapeutics, vol. 16, no. 6, pp. 641-648, 2016.

[13] W. Han and H.-M. Wang, "Refractory diarrhea: A paraneoplastic syndrome of neuroblastoma," World Journal of Gastroenterology, vol. 21, no. 25, pp. 7929-7932, 2015.

[14] K. A. Schwartz, S. J. Slichter, and L. A. Harker, "Immunemediated platelet destruction and thrombocytopenia in patients with solid tumours," British Journal of Haematology, vol. 51, no. 1, pp. 17-24, 1982.

[15] A. W. Berkman and T. Kickler, "Platelet-associated IgG in patients with lymphoma," Blood, vol. 63, no. 4, pp. 944-948, 1984. 


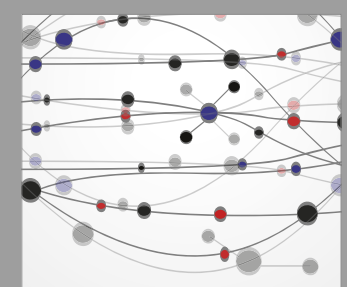

The Scientific World Journal
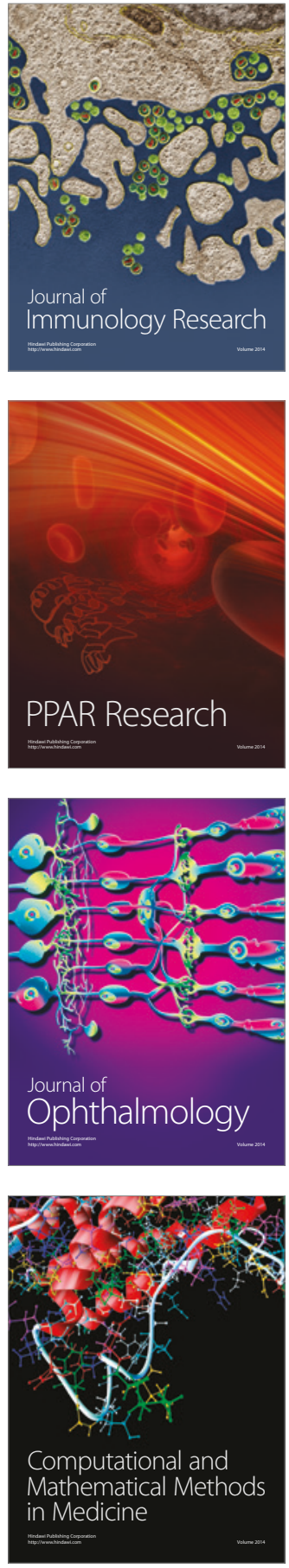

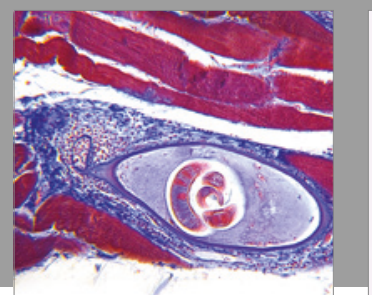

Gastroenterology Research and Practice
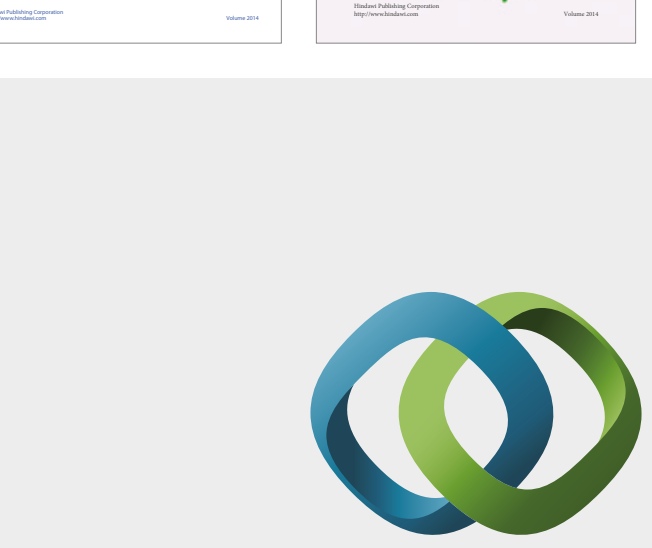

\section{Hindawi}

Submit your manuscripts at

https://www.hindawi.com
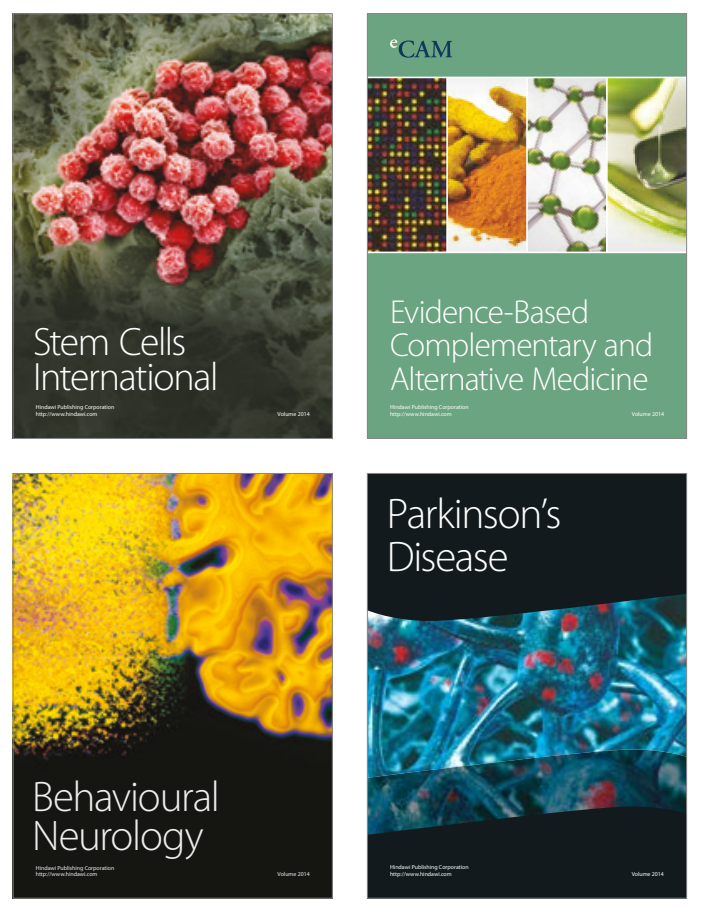
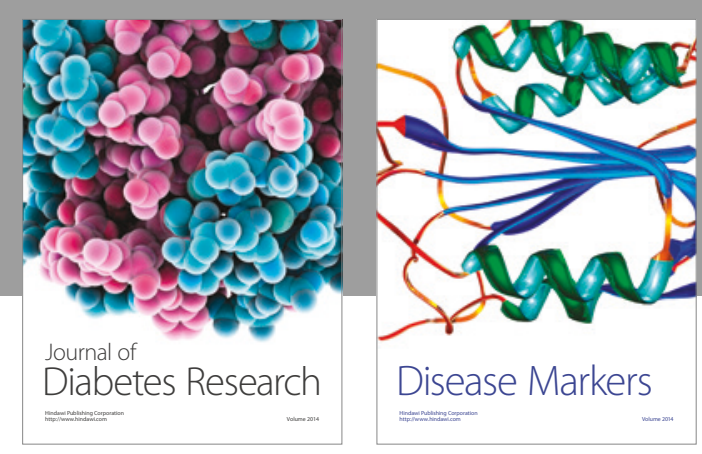

Disease Markers
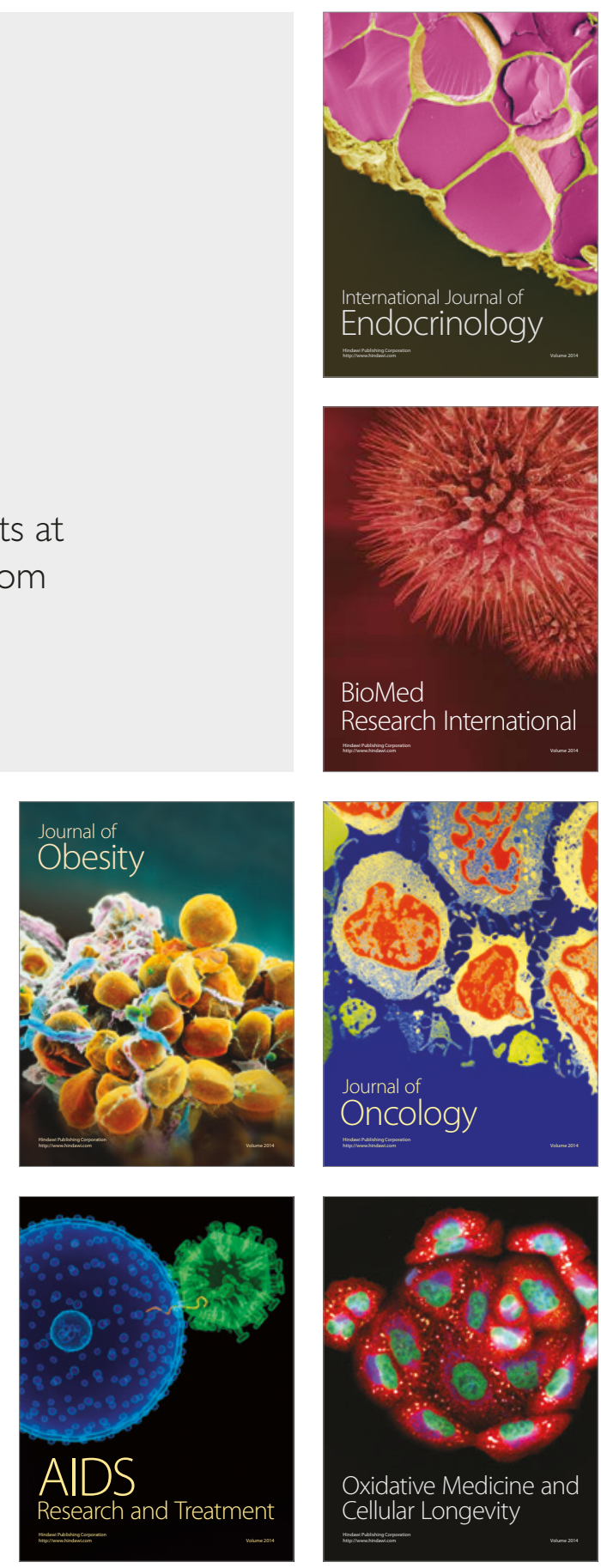